\title{
Securing Healthcare Information in Cloud
}

\author{
S. Aarif Ahamed, V. Ashok Kumar
}

\begin{abstract}
Due to massive growth of technologies in past few years healthcare professionals used to store their patients information in the form of Electronic Medical Record (EMR) which includes huge multimedia medical big data (MDB) such as $X$-rays, Ultrasounds, CT Scan, MRI Reports etc., in healthcare clouds. By means of healthcare cloud, a healthcare professional can be able to access the patient information in an efficient manner when the patient moves from one hospital to another. Even though, healthcare cloud provides easy access to patient data, security issues may happen that may be a leakage of data, privacy \& lack of transparency, etc. The main scope of this study paper is to discuss about various terminology, technology and techniques about the latest methods of cloud computing used in healthcare maintenance system.
\end{abstract}

Keywords : Electronic Medical Record (EMR), Healthcare Cloud, Multimedia Medical Big Data (MDB).

\section{INTRODUCTION}

Cloud Computing is current emerging techniques that provides computing (Processor and Memory) and cloud (Anywhere Any Time) resources on demand with the principles of pay as you go that is pay for the service which we are using. It may include servers, networks, applications and storage as well as service. Cloud Computing enables us to store, process and access the data on remote server via internet.

Cloud Computing serves in three different categories, which are:

- Infrastructure as a Service - Here the service provider gives the complete infrastructure to the users without mentioning the hardware and middleware running behind the server.

- Platform as a Service - Here the user can develop the

- own project with the use of inbuilt services in the platform without any installation.

- Software as a Service - Here the user can access the

- online applications without any installation in computer, access everything via internet.

Revised Manuscript Received on July 22, 2019.

S. Aarif Ahamed, Assistant professor, Vel Tech Rangarajan Dr. Sagunthala R\&D Institute of Science \& Technology, Avadi, Chennai. aarifahamed10@gmail.com,

V. Ashok Kumar, Assistant professor, Vel Tech Rangarajan Dr. Sagunthala R\&D Institute of Science \& Technology, Avadi, Chennai. ashokvijay872@gmail.com

\section{Cloud Computing service models are:}

1. Public cloud - here the resources are available to the general public and it is sharable storage. This type of Cloud is easy and inexpensive because hardware, application and bandwidth costs are covered by the provider.

2. Private cloud - It is used by an organization and it is dedicated storage. Private Cloud gives companies direct control over their data.

3. Community cloud - in which infrastructure is shared with specific community managed by either internally or externally.

4. Hybrid cloud - uses a mix of private cloud and public cloud services.

\section{BACKGROUND \& ARCHITECTURE}

A Security a Security Model for Preserving the Privacy of Medical Big Data in a Healthcare Cloud Using a Fog Computing Facility with Pairing-Based Cryptography

Because of enormous development of advancements in recent years human services experts used to store their patient's data as Electronic Medical Record (EMR) which incorporates immense interactive media therapeutic huge information (MDB, for example, X-beams, Ultrasounds, CT Scan, MRI Reports and so on., in social insurance mists. By methods for social insurance cloud, a human services proficient can have the option to get to the patient data in an effective way when the patient moves starting with one medical clinic then onto the next. Despite the fact that, medicinal services cloud gives simple access to persistent information, security issues may happen that might be a spillage of information, protection and absence of straightforwardness, and so on. So as to explain these security issues, the proposed framework uses haze processing strategy i.e., making a Decoy framework that shrouds the genuine information of client.

At the point when a client needs to transfer Multimedia Big Data (MDB), he/she needs to logon to medicinal services cloud by giving vital subtleties. When a client makes his/her record in the human services cloud, they can transfer their MDB documents. At the point when the client transfers the MDB document, utilizing haze figuring offices and distraction strategies, a DMBD ie. Bait Multimedia Big Data record is made all the while and set adjacent to the OMBD ie. Unique Multimedia Big Data 
record so as to shroud genuine information and makes aggressor to believe that the person got to the Original Data.

The OMBD will be put away in a scrambled organization. With the assistance of Public Key Generator (PKG), a mystery key produced. By utilizing these key, the client needs to scramble the OMBD record and store the encoded document in the cloud. The beneath figure 2.1, speak to the work process of this framework [1].

At the point when a cloud client needs to bring the MBD record, the person needs to login to the cloud. The framework checks whether the client is approved client or not. On the off chance that the client is approved the framework will permit to get to the MBD documents. If not, the framework confines the client to get to the MBD document. In an extraordinary case, there are a few clients called inside assailants who access the framework as approved client by giving substantial login subtleties. When the client/insider aggressor gets to the cloud, DMBD will be shown first.

For approved client after confirmation, unique OMBD is appeared. The confirmation is finished by sending OTP message to the approved client. Subsequently, on the off chance that he/she gives right OTP, that implies he/she might be an approved client, so this framework enable the client to get to the Original Multimedia Medical Big Data which is put away on cloud server.

If not, this framework identifies that client isn't approved client and naturally logout's by sending SMS containing the assailants information like IP address, Date and Access Time to approved client.

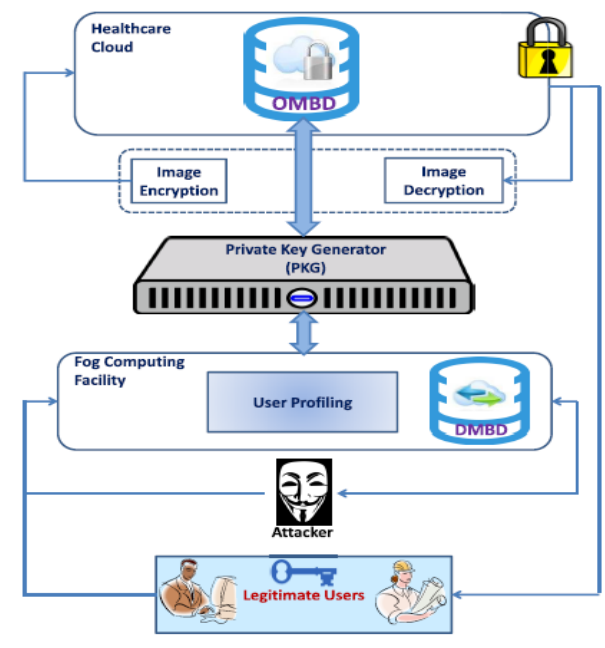

Figure 2.1: Flow of the Fog Computing Method with Pairing-Based Cryptography

Biometric Based Signature Authentication Scheme for Cloud Healthcare Data Security

This paper deals with Biometric signature based authentication system with Neural Network to improve health care security. Biometric authentication has been increasing nowadays for monitoring the physical presence in many industries, now it is extended to medical field for health care management. The growing demand leads to reduce the fraudulent and also to improve the quality of privacy as well as safety. The below figure 2.2, represent the workflow of this system [2].

This system stores medical Big Data in cloud environment with a signature to ensure security. This method includes signature processing and quality checking, Map Reduce technique is used for data storage and neural network $(\mathrm{NN})$ is used for verifying the biometric signature. This method produces high accuracy and better throughput thus reduces the overall cost of the system. Here the biometric signature from healthcare providers, doctors / patients are obtained via smart phones. Capturing the signature from user is thoroughly examined with quality checks for confirm the quality of signature and processed with MapReduce framework. When a user requested for access to particular medical Big Data they must provide biometric signature for verification if the biometric signature is matched with the stored content than the user is provided with access otherwise the user must enroll themselves.

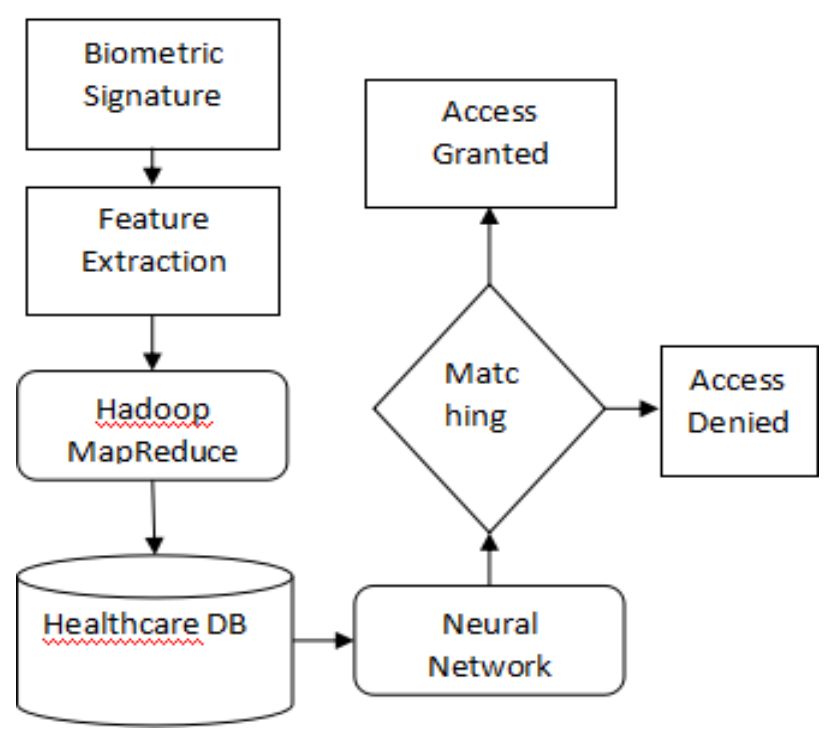

Figure 2.2 : Flow Chart of Signature based authentication system.

This system mainly using two phases called enrollment phase ( for enrollment of new user) and authentication phase ( for verification). In first phase, the biometric signature is acquired using suitable reader then processing is done with extracted features using Hadoop MapReduce framework followed by second phase, here the system checks for uniqueness of signature.

\section{Intelligent Attribute Based Encryption (IABE) Mechanism for Health Records in Cloud}

This paper deals with attribute based encryption method which is one flavor of public key cryptographic concept in which secret key \& cipher text depends upon certain attributes that belongs to users. Here the decryption is done only if the user key matches with the attributes of encrypted text. This paper introduces an agent that agent will act as 
intermediary between Cloud server and users. Here owner is the client who is going to upload the medical record and also key both submitted to the agent. This will generate new key called control key then encryption is done by applying both keys with timestamp. If the time span elapses, the control key and information key is removed from agent. This will reduce the attacks. The below figure 2.3 , represent the workflow of this system [3].

- Input for Encryption: Public Health Record Key

- Output after Encryption: Cipher Text

- Input for Decryption: Control key

- Output after Decryption: Plain text

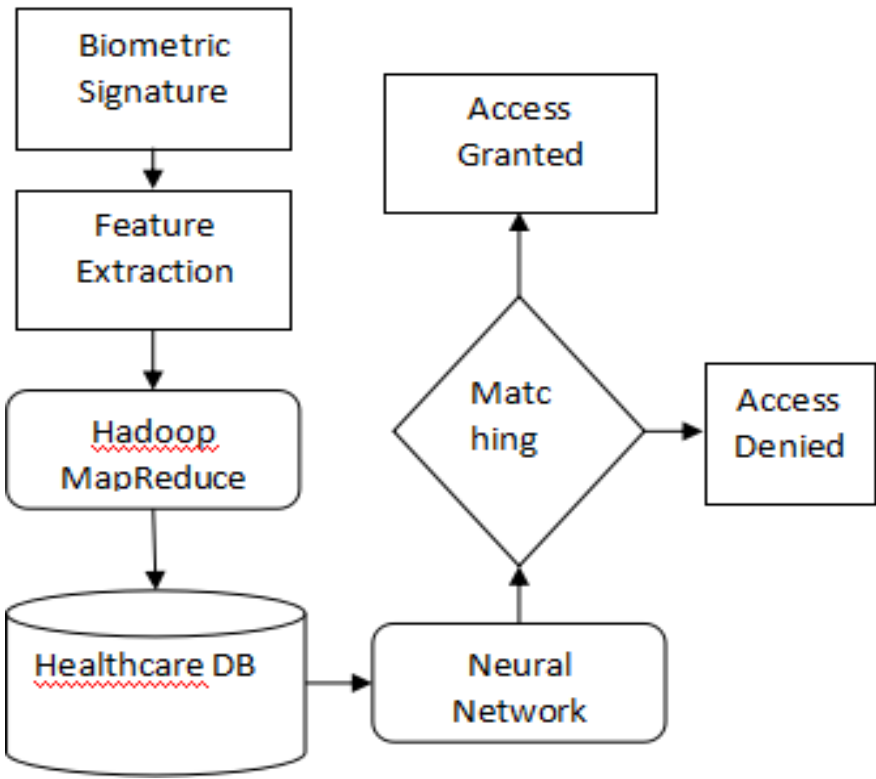

Figure 2.3: Flow Chart of Attribute based authentication system

It is very difficult to decode with control key alone because this cipher text is time stamped, all the keys including control key, information key, PHR keys are removed once the time stamp running out. This method will guarantees timely delivery and security of medical record against the attacks in cloud.

Securing Healthcare Information over Cloud Using Hybrid Approach

K. J. Modi and N. Kapadia [4] have proposed a framework that gives a solution to secure the healthcare information in cloud. This method supports hybrid approach with the combination of linear network coding and re-encryption techniques. This system uses ElGamal encryption technique which is an asymmetric key encryption algorithm. Linear Network encoding reduces network delays and increases the throughput and makes it as more robust and reliable and thus improves the security of the network. When coming to the another hand of proxy re-encryption, it converts the cipher text into some form that can be decoded by private key of receiver without the use of private key of sender. This method is something like A sends a encoded mail to B without sharing private key. Here, A uses proxy re-encryption technique to re-encode an email into some form that B can decode by using own private key.

Data sharing is done through the following steps: if any of the cloud users like a doctor or nurse wants to get the patient data then he/she gives a request. Here the private key is divided into two parts (say $\operatorname{Pr} 3$ and $\operatorname{Pr} 4$ ). One part of private key (Pr3) will sent to storage unit that will be stored as meta data the proxy will converts into some form and the remaining part $(\operatorname{Pr} 4)$ is send to the user who requests. After receiving the $\operatorname{Pr} 4$, the doctor will download the any file from cloud and decrypt with Pr4 to get the original Data.

\section{PERFORMANCE COMPARISON}

\section{Performance Analysis of Paper I}

Based upon the comparison with DES, AES, Triple DES and Blow Fish algorithms, the first paper achieves the better performance (i.e blowfish algorithm) than other encryption methods in terms of block size, number of rounds, key length ,encryption and decryption time, power consumption, memory usage and confidentiality. So this paper suggested blow fish algorithm for the better result in terms of power and memory usage followed by key exchange. Key Exchange is done through ECC algorithm which is more secured and ensuring that connection made between appropriate sender and intended receiver

Table 1: Key Size Comparison between RSA algorithm and ECC algorithm

\begin{tabular}{|c|c|c|}
\hline $\begin{array}{c}\text { Security Level } \\
\text { (in Bits) }\end{array}$ & $\begin{array}{c}\text { RSA Key Size } \\
\text { ( in Bits) }\end{array}$ & $\begin{array}{c}\text { ECC Key } \\
\text { Size (in Bits) }\end{array}$ \\
\hline 80 & 1024 & 160 \\
\hline 128 & 3072 & 256 \\
\hline 160 & 4096 & 320 \\
\hline 256 & 15360 & 521 \\
\hline
\end{tabular}

This methodology involves 1 elliptic curve multiplication and addition operation, 1 hash evaluation on $\mathrm{H} 1$, and three on $\mathrm{H} 2$ followed by 1 random number generation. The following graph represents the computational cost for each methods and this method. Cost wise this method may be preferable.

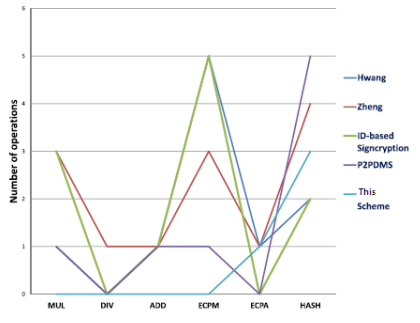

Figure 3.1: Comparison between different schemes with Paring based Cryptography 


\section{Performance Analysis of Paper II}

This paper researched with 300 signatures as a sample from patient as well as staffs in hospital. The collected signatures

were processed after quality check. The results produce higher sensitivity and accuracy with medical records. Comparisons are made with SVM's Average IRF, SVM's Genetic Algorithm and SVM's Fuzzy Logics. Among different classifiers, this method produces better accuracy, precision and sensitivity. Accuracy wise this method may be preferable.

\section{Table 2: Performance comparison}

\begin{tabular}{|c|c|c|c|}
\hline Algorithms & Accuracy & Precision & Sensitivity \\
\hline This Method & 0.996 & 0.825 & 0.943 \\
\hline AIRF & 0.995 & 0.825 & 0.943 \\
\hline GA & 0.996 & 0.869 & 0.935 \\
\hline Fuzzy & 0.996 & 0.869 & 0.936 \\
\hline
\end{tabular}

\section{Performance Analysis of Paper III}

This Paper produces the result of faster time in encryption but the drawback of this method is it takes more time in decryption. For 30 attributes, it takes $150 \mathrm{~ms}$ for encrypting but it takes $250 \mathrm{~ms}$ for decrypting. The $\mathrm{X}$ and $\mathrm{Y}$ axis indicates number of attributes and time respectively. If the time is not a concern means this method may be preferable.

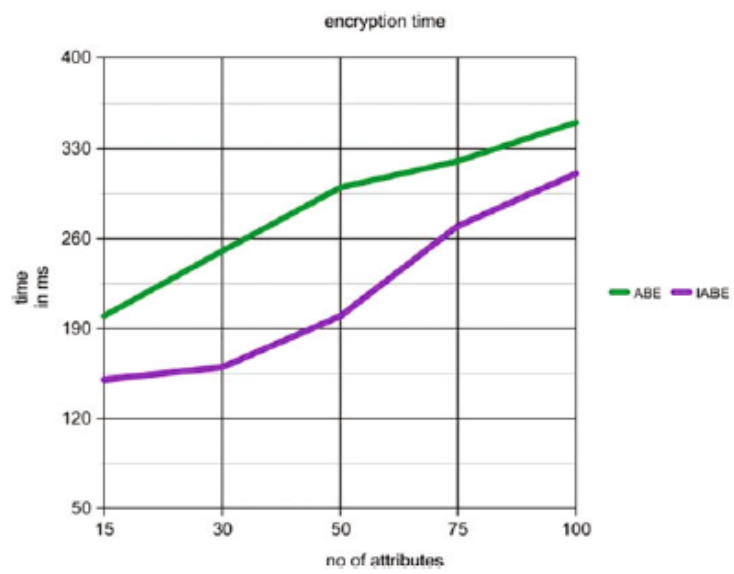

Figure 3.2: Comparison Chart for Encryption

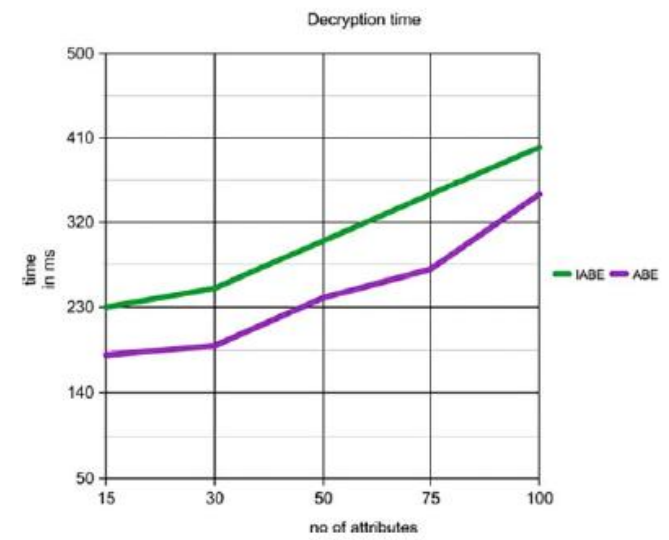

Figure 3.3: Comparison Chart for Decryption

\section{Performance Analysis of Paper IV}

This paper did experiment with package of S3 provided by Amazon web services. With the support of Eclipse Kepler version 4.3, the buckets are created and applied the proposed algorithm over a file then metadata is added to the file containing partial decrypted key. Experiment is conducted for 6 nodes. Following graph shows reliability level with the Linear Network Encoding approach. Using this approach we can recover more data when compare to other approaches. This paper will be referred for textual data but not for the medical data's.

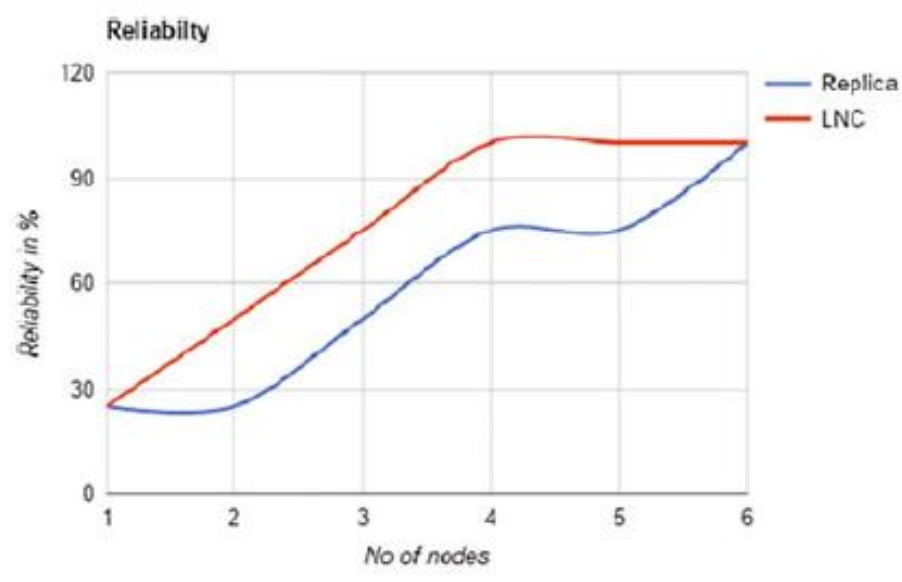

Figure 3.4: Comparison Chart of Reliability

\section{CHALLENGES \& OPEN RESEARCH ISSUES}

While studying the above papers still lot of lagging in the security while storing and accessing the data to the doctor from cloud for further processing. In first paper new methodology of decoy system is introduced, for every data updates it takes more time to create fake data to confuse the attacker while looking at the second paper, if anyone get the biometric signature means it is very easy to breach the security for that artificial intelligence to be included so that

the real data's must be captured and process simultaneously. In third paper we assume that no one hacks the agent. If someone hacks the agent means it is very easy to trace the control key and information key and this leads to a chance of data alteration. Last paper deals only with textual data when it is moved to digital data means need to concentrate on lossless retrieval of image otherwise this method leads to failure.

\section{RESULT AND CONCLUSION}

The ultimate aim of the study paper is secure Multimedia Medical Big Data files in healthcare system maintained in cloud using different approaches. Cloud computing is more demand and trending nowadays for elasticity, reliability, scalability of services. So only the need is increased to medical field also to store large set of data for processing and accessing the patient details at anywhere anytime. Many enhancements are made with existing solutions but still the security problem remains same. New solutions are needed to meet 
the demand of cloud in medical field like block chain technology to fix the block once it is created with multi authority to authorize the data's.

\section{REFERENCES}

1. Hadeal Abdulaziz Al Hamid, Sk Md Mizanur Rahman, M. Shamim Hossain, Ahmad Almogren and Atif Alamri,“ A Security Model for Preserving the Privacy of Medical Big Data in a Healthcare Cloud Using a Fog Computing Facility With Pairing-Based Cryptography", VOLUME 5, 2017 IEEE.

2. Gunasekar Thangarasu, P. D. D. Dominic, Kayalvizhi Subramanian and and Sajitha Smiley, " Biometric Based Signature Authentication Scheme for Cloud Healthcare Data Security," in IRICT 2018, AISC 843, pp. 557-565, 2019.

3. Ranjith Kumar Vollala and L. Venkateswara Reddy, "Intelligent Attribute Based Encryption (IABE) Mechanism for Health Records in Cloud", Computing, Communication and Signal Processing, Advances in Intelligent Systems and Computing 810, pp. 197 - 204, https://doi.org/10.1007/978-981-13-1513-8_21

4. Kirit J. Modi and Nirali Kapadia, "Securing Healthcare Information over Cloud Using Hybrid Approach", Progress in Advanced Computing and Intelligent Engineering, Advances in Intelligent Systems and Computing 714, pp. $63-73$ https://doi.org/10.1007/978-981-13-0224-4_6.

5. https://en.wikipedia.org 